\title{
The Effect of Resistance Training on Telomere Length in Women Recovering from Breast Cancer
}

\author{
Amanda D. Hagstrom ${ }^{(D)}$ and Joshua Denham \\ School of Science and Technology, University of New England, Armidale 2350, Australia; \\ ahagstro@une.edu.au (A.D.H.); jdenham2@une.edu.au (J.D.); Tel.: +61-2-6773-3166 (A.D.H)
}

Received: 10 January 2018; Accepted: 23 January 2018; Published: 26 January 2018

\begin{abstract}
The purpose of this exploratory analysis was to examine the effects of resistance training (RT) on leukocyte telomere length in breast cancer survivors. Samples from 33 breast cancer survivors were analysed following a 16-week RT or usual care intervention (RT: $n=19$, control: $n=14$ ). The RT group performed supervised exercise three times per week. Leukocyte telomere length was assessed before and after training using quantitative PCR. Changes in telomere length were analysed using ANCOVA, with the change score being the dependent variable, and the baseline value the covariate. Effect sizes (ES) were calculated via partial eta squared. We found a non-statistically significant change in telomere length, and a small associated ES in the RT group compared to the control group for change in telomere length $(p=0.78, \mathrm{ES}=0.003)$. Our exploratory study demonstrates that a 16-week RT intervention does not have a significant effect on telomere length in women recovering from breast cancer. Therefore, RT is a safe intervention that does not accelerate biological ageing.
\end{abstract}

Keywords: resistance training; telomere; breast cancer

\section{Introduction}

Breast cancer is the second most commonly diagnosed cancer in women, accounting for one in three cancer diagnoses [1]. Cancer accelerates biological ageing through cellular senescence and genomic instability and is typically associated with short telomeres [2,3]. Telomere length is a marker of biological age [4], with shorter telomere length being associated with older ages. Recent data indicates that telomeres may be lengthened rather than maintained through healthy lifestyle interventions [5-7]. As such, research examining telomere length may provide a foundation for the identification of health-related behaviours that may enhance rehabilitation in cancer survivors [8].

While lifestyle interventions have been successful in altering telomere length, the long-term relationship between exercise and telomeres remains unclear. Frequent participation in exercise and a superior cardiorespiratory fitness is associated with longer telomeres [9]. In breast cancer survivors, a lack of self-reported physical activity (PA) is linked to shortened peripheral blood mononuclear cell telomeres $[8,10]$. Further, those individuals reporting participation in moderate to vigorous PA display an approximate $4.5 \%$ great average telomere length, corresponding to a mean difference of $270 \mathrm{bp}$ [8].

The impacts of resistance training (RT) on telomere dynamics are less understood. A cross-sectional study [11] compared the skeletal muscle telomere length of seven power lifters with seven healthy active subjects with no history of strength training. These researchers found a non-significant tendency for longer telomeres in the strength-trained powerlifting group $(p=0.07)$. Conversely, others examined telomere length changes following a six-month resistance training intervention in elderly subjects residing in a nursing home and observed no differences between experimental or control groups [12]. Further, a 16-week study examining the effect of three times weekly RT in a group of women with polycystic ovarian syndrome found telomere shortening in both the experimental and control group [13]. Therefore, whether RT maintains or accelerates biological ageing through telomere length regulation remains unclear. 
Exercise is now part of a multidisciplinary rehabilitation approach for cancer survivors. Resistance training has grown in popularity in recent years due to its ability to improve a variety of factors (e.g., inflammatory markers [14], muscular strength, endurance, and range of motion [15,16]). Therefore, as part of a randomised controlled trial designed around immunological outcomes, this study aimed to examine the effect of resistance training on leukocyte telomere length in a cohort of breast cancer survivors. We hypothesised that a 16-week training intervention would not accelerate biological ageing in the form of telomere shortening.

\section{Materials and Methods}

\subsection{Approach to the Problem}

This investigation involved a controlled, prospective analysis of leukocyte telomere length after 16 weeks of RT in women recovering from breast cancer. This study was designed to explore the potential for RT to influence leukocyte telomere length (dependent variable) whilst controlling for known biological traits related to telomere biology (independent variables-age, weight, etc.).

\subsection{Subjects}

This parallel-arm randomised controlled clinical trial was designed around primary immunological outcomes [14] comparing breast cancer survivors assigned to a 16 week RT group $(n=19)$ with those assigned to a usual care control group $(n=14)$. Primary findings from this trial relating to immunological outcomes, muscular strength, quality of life, and fatigue have been previously reported $[14,17,18]$. Outcome measures were collected at week 0 and 17 . This study was a post hoc analysis examining the effect of RT on telomere length in this cohort of women. The study was approved by Western Sydney University (WSU) Human Research Ethics (Approval Number: H9427, 23/03/2012). The subsequent post-hoc study was approved by the University of New England Human Research Ethics Committee (HE16-224, 10/10/2016). The telomere assays took place at the University of New England in December 2016. The clinical trial was registered with the Australian and New Zealand clinical trials registry (ANZCTR \#12612000346875). Informed consent was obtained from all participants.

The recruitment of participants, eligibility, exclusion criteria, and randomisation are described elsewhere $[14,17,18]$. To participate in the initial study, participants must have had a history of histologically confirmed stage I to IIIA breast cancer with no evidence of recurrent disease; age 18-70 years; completed surgery, radiotherapy, and/or chemotherapy; and sedentary ( $<30 \mathrm{~min}$ of structured, continuous moderate-intensity exercise, three times per week, and no current resistance training). Survivors were stratified based on age ( $<50$ or $>50$ years) and current use of hormone therapy (Yes; No) and then randomised to a control or RT group. For this post hoc analysis, an additional inclusion criterion was that the participants must have completed the intervention as well as both baseline and follow-up testing sessions.

\subsection{Procedures}

\subsubsection{Resistance Training Intervention and Measurement of Muscular Strength}

As methods and findings relating to the testing and training of muscular strength have been previously published $[14,17,18]$ we have not included the details here. These outcomes were used for correlational purposes only. However, as demonstrating the efficacy of the strength training program is important for the primary outcomes of this paper (telomere length), and to aid in understanding, we have briefly detailed the methods previously utilised here. Briefly, lower body muscular strength was assessed via a 1 repetition maximum (RM) leg press, while upper body muscular strength was assessed unilaterally via an isometric chest press. In summary, supervised RT was performed three times per week for $60 \mathrm{~min}$ per session. The exercise load was set at an 8 repetition maximum (RM), 
meaning that the participants lifted a weight that they could successfully lift eight times. Loads were increased when the participant gained the ability to lift 10 repetitions at the same weight prior to muscular failure. The first eight weeks of the exercise program consisted primarily of machine-based exercises (leg extension, leg curl or Romanian deadlift, lat pulldown, machine bench press, seated row, back extension, prone hold or sit ups). The final eight weeks of the program predominantly focused on free weights (barbell squat, deadlift, bench press, leg press, barbell bent over row, assisted chin up). Each exercise was performed for three sets.

\subsubsection{Blood Markers and Telomere Length}

Individuals involved in the analyses of blood markers and telomere length quantification were blinded to the group assignment. Venous blood was donated in an overnight fasted state, collected into heparin tubes and frozen at $-20^{\circ} \mathrm{C}$ prior to DNA extraction. DNA extraction, yield, and purity as well as telomere length were assessed as previously described [19,20]. Briefly, frozen blood samples were thawed on ice and DNA was extracted from buffer coat leukocytes using the Purelink Genomic DNA Mini Kit following the manufacturer's guidelines (Thermo Fisher Scientific, Waltham, MA, USA). As DNA purity could influence telomere length assayed by qPCR [21], purity was assessed on the NanoDrop 8000 Spectrophotometer (Thermo Fisher Scientific). All samples showed acceptable purity with $260 / 280$ and $260 / 230$ ratios of $1.72 \pm 0.14$ and $1.26 \pm 0.34$, respectively. Telomere length was quantified using qPCR by comparing the telomere (T) repeat sequence to a single (S) copy gene (36B4). Experimental and control samples were run in triplicate on the same 384-well plates for telomere and 36B4 primer sets. Experiments comprised of DNA template or water (for no-template controls), and either $300 \mathrm{nM}$ of forward and reverse primer for the telomere sequence or $300 \mathrm{nM}$ of the forward and $500 \mathrm{nM}$ of the reverse primer for the 36B4 sequence, were run on a Bio-Rad Thermocycler (CFX96 Touch Real-Time PCR Detection System, Bio-Rad, Hercules, CA, USA). Primer sets and thermocycling conditions were identical to those previously described [19]. Telomere length was expressed in arbitrary units as a $\mathrm{T} / \mathrm{S}$ ratio, relative to a healthy 30 -year-old woman using the $2^{-(\Delta-\Delta \mathrm{Ct})}$ method.

\subsection{Statistical Analysis}

A power calculation was not performed for this post hoc analysis. Sample size estimates were initially based upon the primary immunological (natural killer cell activity, NKCA) outcomes from a previous study [14]. Primary analysis was via a per-protocol approach. Baseline characteristics were compared between groups using independent $t$-tests for continuous variables and chi-square tests for categorical variables. Data were presented as the mean and SD with effect sizes (ES). Primary analysis compared change in telomere length using an ANCOVA where the baseline value was the covariate. Paired samples $t$-tests were also performed to establish within-group changes in telomere length. Statistical significance was set at $p<0.05$. Partial $\eta^{2}$ was used to measure ES where 0.01, 0.06, and 0.14 were considered small, medium, and large, respectively. Relationships between telomere length and variables that we have previously shown to demonstrate significant changes were analysed using Pearson correlation coefficients $(r)$.

\section{Results}

\subsection{Baseline Characteristics}

There were no significant differences between groups at baseline for age or treatment regimens, including chemotherapy, radiotherapy, and hormone therapy (all $p>0.05$ ) (Table 1). 
Table 1. Clinical characteristics at baseline.

\begin{tabular}{cccc}
\hline Variable & RT Group $(\boldsymbol{n}=\mathbf{1 9 )}$ & Control Group $(\boldsymbol{n}=\mathbf{1 4})$ & $\boldsymbol{p}$-Value \\
\hline Age (years) & $50.8 \pm 8.3$ & $52.1 \pm 8.5$ & 0.67 \\
Body mass index $(\mathrm{BMI})\left(\mathrm{kg} / \mathrm{m}^{2}\right)$ & $27.7 \pm 4.3$ & $30.3 \pm 6.3$ & 0.23 \\
Weight $(\mathrm{kg})$ & $72.7 \pm 9.3$ & $80.1 \pm 16.7$ & 0.15 \\
Time since treatment (months) & $8.5 \pm 8.8$ & $8.4 \pm 10.5$ & 0.99 \\
Chemotherapy $(n)$ & 16 & 12 & 0.90 \\
Radiotherapy $(n)$ & 18 & 13 & 0.82 \\
Hormone therapy $(n)$ & 16 & 13 & 0.45 \\
\hline
\end{tabular}

Data are from two-tailed independent samples $t$-tests or $\mathrm{Chi}^{2}$ tests and are expressed as mean $\pm \mathrm{SD}$ or frequencies.

\subsection{Adherence and Adverse Events}

Adherence and adverse event data has previously been reported for the entire cohort [14]. Briefly, no adverse events occurred during this study. Adherence to the exercise intervention for this subset of the cohort was $85.9 \%$ attendance at exercise sessions (range: $46.8-100 \%$ ).

\subsection{Telomere Length}

There were no statistically significant differences in leukocyte telomere length at baseline between groups (mean T/S ratio $\pm \mathrm{SD}$ : RT, $0.43 \pm 0.11$; control, $0.37 \pm 0.10, p=0.11$ ). There was no significant difference in change in telomere length between groups $(p=0.78, \mathrm{ES}=0.003$, Figure 1$)$. While the RT group telomeres were relatively unchanged after the 16-week intervention $(0.43 \pm 0.11$ to $0.44 \pm 0.09, p=0.82)$, the control group showed a modest increase in telomere length $(0.37 \pm 0.10$ to $0.42 \pm 0.13, p=0.13)$. Thirteen $(39.4 \%)$ and $20(60.6 \%)$ participants experienced telomere shortening and lengthening, respectively.

A

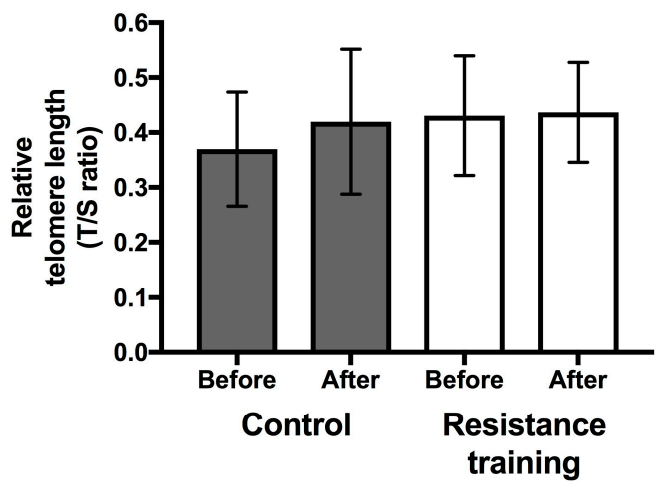

B

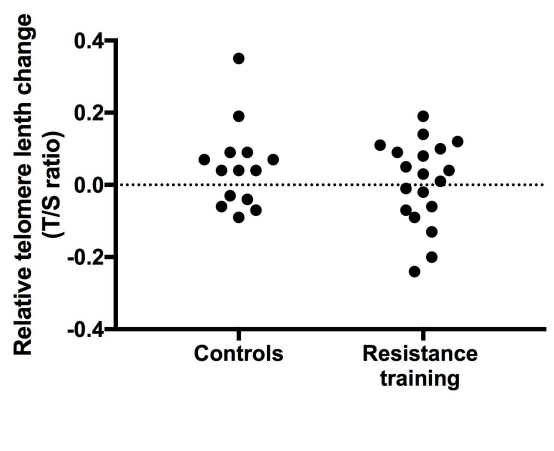

Figure 1. Leukocyte telomere length before and after 16 weeks of resistance training in breast cancer survivors. Mean telomere length (mean $\pm \mathrm{SD},(\mathbf{A})$ ) and telomere length change $(\mathbf{B})$ are presented in arbitrary units relative to the T/S ratio of an apparently healthy 30-year-old woman (RT: $n=19$, control: $n=14$ ). DNA purity was acceptable with $260 / 280$ and $260 / 230$ ratios of $1.72 \pm 0.14$ and $1.26 \pm 0.34$, respectively. Experiments were repeated and demonstrated excellent reliability for telomere $\left(R^{2}=0.94\right)$ and $36 B 4\left(R^{2}=0.97\right)$ primer sets. The intra- and inter-assay co-efficient of variation $(\mathrm{CV})$ for experimental samples were acceptable (telomere $C V \pm S D: 1.45 \pm 3.49 \%$ and $1.62 \pm 3.28 \%$; $36 B 4$ $\mathrm{CV} \pm \mathrm{SD}: 0.53 \pm 2.08 \%$ and $1.34 \pm 2.16 \%$, respectively). $\mathrm{T}$, telomere; $\mathrm{S}$, single copy gene (36B4).

\subsection{Response to the Resistance Training Intervention}

Within the RT group, there was no significant change in body mass index (BMI) (27.7 \pm 4.3 to $\left.27.8 \pm 3.9 \mathrm{~kg} / \mathrm{m}^{2}, p=0.48\right)$ or body weight $(72.7 \pm 9.3$ to $73.0 \pm 8.7 \mathrm{~kg}, p=0.59)$ over the course of 
the intervention. There was also no significant change with in the control group in BMI ( $30.2 \pm 6.2$ to $\left.30.5 \pm 6.6 \mathrm{~kg} / \mathrm{m}^{2}, p=0.42\right)$ or body weight $(80.1 \pm 16.7$ to $80.8 \pm 18.3 \mathrm{~kg}, p=0.38)$ over the course of the intervention. There was no significant difference in change in BMI $\left(\mathrm{kg} / \mathrm{m}^{2}\right)$ or body weight $(\mathrm{kg})$ between the two groups ( $p=0.75, \mathrm{ES}=0.004 ; p=0.87$, $\mathrm{ES}=0.001$ ).

In the subset of participants included in this post hoc analysis, muscular strength (mean percent change \pm SD) improved in the RT group in all measurements (Surgical arm, $28.97 \pm 22.68 \%$; other arm, $34.50 \pm 27.27 \%$; lower body (via leg press), $42.55 \pm 19.85 \%$ ). There was a significant within-group difference over time $(p<0.001)$ for all variables in the RT group. In comparison, the muscular strength of those assigned to the control group did not change (Surgical arm; $-5.38 \pm 17.86 \%$, other arm; $1.03 \pm 17.02 \%$, lower body; $-6.54 \pm 20.09 \%$ ). There were no within-group differences over time in the control group ( $p=0.28, p=0.82$, and $p=0.28$, respectively).

\subsection{Relationships between Telomere Length and Muscular Strength}

No significant correlations were found between changes in muscular strength and telomere length (Table 2).

Table 2. Linear correlations between change in muscular strength and telomere length.

\begin{tabular}{ccc}
\hline Variable (\%) & $r$-Value & $p$-Value \\
\hline Upper body strength change in the surgical arm & 0.035 & 0.85 \\
Upper body strength change in the other arm & 0.05 & 0.78 \\
Lower body strength change & -0.02 & 0.93 \\
\hline Data are from two-tailed Pearson's correlation $(n=28-33)$.
\end{tabular}

\section{Discussion}

We are the first to examine the longitudinal effect of RT on telomere length in breast cancer survivors. Our hypothesis was supported, and the null hypothesis was rejected as we observed no statistically significant difference in telomere length change between the RT and control groups. Additionally, strength was not associated with telomere length.

Whilst multiple studies have demonstrated that those individuals who are physically active possessed longer leukocyte telomeres compared to less active controls [9], longitudinal intervention-based research is lacking. In one of the few longitudinal studies, a four-month RT intervention in women with polycystic ovary syndrome, a shortening of telomeres was demonstrated [13]. In our study, $56 \%$ and $44 \%$ of participants experienced telomere shortening and lengthening, respectively. Similarly, in a longitudinal study examining telomere length in breast cancer survivors it was found that $32 \%$ of participants exhibited a lengthening of telomeres, whereas $68 \%$ showed shortening [22]. We also observed variation in telomere length changes after the intervention, such that telomere attrition was observed in $39 \%$ of patients (61\% showed telomere extension). These studies highlight the large inter-individual variation in telomere dynamics and in response to resistance exercise. Indeed, whilst the large inter-individual responses to telomere length in the RT and control groups could be responsible for the lack of statistically significant changes, an alternate interpretation is that RT does not impact telomere length over a relatively short period. However, it is possible that longer interventions may uncover the potential benefit of RT on telomere dynamics and biological ageing.

As telomere length is affected by multiple environmental factors including psychological stress, nutrition, body composition, sleep quality, and ageing [23], it may be difficult to deduce the isolated effects of exercise without controlling for these variables. In our study, all but four participants were on some form of hormone-modulating medication, which may partially explain the lack of consistent telomere length change observed in both groups following our intervention. Although not statistically significant, there was a large difference in body weight between cases and controls at baseline. This is a limitation as there seems to be a link between obesity and telomere shortening, particularly in 
women $[24,25]$. In addition, due to the nature of the study we were unable to control for all chronic diseases (e.g., hypertension), as such there is a possibility that underlying disease may have influenced our findings. We did, however, use an established method for quantifying average telomere length. An assessment of the frequency of short and long telomeres using quantitative fluorescent in situ hybridization or Southern Blot was not possible due to sample storage and processing restrictions. These methods should be considered in future analyses to quantify frequencies of minimum and maximum telomere length. Although telomere length was not significantly different between groups in our study, it is possible that the small sample size and duration of the exercise intervention may have influenced our findings. Therefore, future research utilising larger samples and longer interventions is warranted.

\section{Conclusions}

In conclusion, we did not observe a marked difference in telomere length amongst breast cancer survivors after 16 weeks of RT. Resistance exercise did not accelerate telomere attrition in breast cancer survivors and the inter-individual responses in telomere dynamics after RT should be explored in larger studies. Therefore, due to the myriad of health benefits resulting from RT in this population, this modality of exercise should still be recommended as part of a multifaceted rehabilitation program.

Acknowledgments: The initial study was covered by a grant from Western Sydney University (WSU). All subsequent analysis undertaken at the University of New England was not covered by any external funding source.

Author Contributions: Amanda D. Hagstrom conceived and designed the original trial. Amanda D. Hagstrom and Joshua Denham conceived the follow-up study. Amanda D. Hagstrom performed the experiments and initial data collection; Joshua Denham conducted all subsequent lab-based telomere analysis. Amanda D. Hagstrom and Joshua Denham analysed and interpreted the data. Amanda D. Hagstrom and Joshua Denham wrote the paper.

Conflicts of Interest: The authors declare no conflict of interest.

\section{References}

1. DeSantis, C.; Ma, J.; Bryan, L.; Jemal, A. Breast cancer statistics, 2013. CA Cancer J. Clin. 2014, 64, 52-62. [CrossRef] [PubMed]

2. Wentzensen, I.M.; Mirabello, L.; Pfeiffer, R.M.; Savage, S.A. The association of telomere length and cancer: A meta-analysis. Cancer Epidemiol. Prev. Biomark. 2011, 20, 1238-1250. [CrossRef] [PubMed]

3. Shen, J.; Terry, M.B.; Gurvich, I.; Liao, Y.; Senie, R.T.; Santella, R.M. Short telomere length and breast cancer risk: A study in sister sets. Cancer Res. 2007, 67, 5538-5544. [CrossRef] [PubMed]

4. Andrews, N.P.; Fujii, H.; Goronzy, J.J.; Weyand, C.M. Telomeres and immunological diseases of aging. Gerontology 2010, 56, 390-403. [CrossRef] [PubMed]

5. Epel, E. How "reversible" is telomeric aging? Cancer Prev. Res. 2012, 5, 1163-1168. [CrossRef] [PubMed]

6. Ornish, D.; Lin, J.; Chan, J.M.; Epel, E.; Kemp, C.; Weidner, G.; Marlin, R.; Frenda, S.J.; Magbanua, M.J.; Daubenmier, J.; et al. Effect of comprehensive lifestyle changes on telomerase activity and telomere length in men with biopsy-proven low-risk prostate cancer: 5-year follow-up of a descriptive pilot study. Lancet Oncol. 2013, 14, 1112-1120. [CrossRef]

7. Puterman, E.; Lin, J.; Krauss, J.; Blackburn, E.H.; Epel, E.S. Determinants of telomere attrition over 1 year in healthy older women: Stress and health behaviors matter. Mol. Psychiatry 2015, 20, 529-535. [CrossRef] [PubMed]

8. Garland, S.N.; Johnson, B.; Palmer, C.; Speck, R.M.; Donelson, M.; Xie, S.X.; DeMichele, A.; Mao, J.J. Physical activity and telomere length in early stage breast cancer survivors. Breast Cancer Res. 2014, 16, 413. [CrossRef] [PubMed]

9. Denham, J.; O'Brien, B.J.; Charchar, F.J. Telomere length maintenance and cardio-metabolic disease prevention through exercise training. Sports Med. 2016, 46, 1213-1237. [CrossRef] [PubMed]

10. Ennour-Idrissi, K.; Tetu, B.; Maunsell, E.; Poirier, B.; Montoni, A.; Rochette, P.J.; Diorio, C. Association of telomere length with breast cancer prognostic factors. PLOS ONE 2016, 11, e0161903. [CrossRef] [PubMed] 
11. Kadi, F.; Ponsot, E.; Piehl-Aulin, K.; Mackey, A.; Kjaer, M.; Oskarsson, E.; Holm, L. The effects of regular strength training on telomere length in human skeletal muscle. Med. Sci. Sports Exerc. 2008, 40, 82-87. [CrossRef] [PubMed]

12. Tosevska, A.; Franzke, B.; Hofmann, M.; Vierheilig, I.; Schober-Halper, B.; Oesen, S.; Neubauer, O.; Wessner, B.; Wagner, K.H. Circulating cell-free DNA, telomere length and bilirubin in the vienna active ageing study: Exploratory analysis of a randomized, controlled trial. Sci. Rep. 2016, 6, 38084. [CrossRef] [PubMed]

13. Miranda-Furtado, C.L.; Ramos, F.K.P.; Kogure, G.S.; Santana-Lemos, B.A.; Ferriani, R.A.; Calado, R.T.; dos Reis, R.M. A nonrandomized trial of progressive resistance training intervention in women with polycystic ovary syndrome and its implications in telomere content. Reprod. Sci. 2016, 23, 644-654. [CrossRef] [PubMed]

14. Hagstrom, A.D.; Marshall, P.W.; Lonsdale, C.; Papalia, S.; Cheema, B.S.; Toben, C.; Baune, B.T.; Fiatarone Singh, M.A.; Green, S. The effect of resistance training on markers of immune function and inflammation in previously sedentary women recovering from breast cancer: A randomized controlled trial. Breast Cancer Res. Treat. 2016, 155, 471-482. [CrossRef] [PubMed]

15. Ohira, T.; Schmitz, K.H.; Ahmed, R.L.; Yee, D. Effects of weight training on quality of life in recent breast cancer survivors: The weight training for breast cancer survivors (WTBS) study. Cancer 2006, 106, 2076-2083. [CrossRef] [PubMed]

16. Cheema, B.; Gaul, C. Full-body exercise training improves fitness and quality of life in survivors of breast cancer. J. Strength Cond. Res. 2006, 20, 14-21. [CrossRef] [PubMed]

17. Hagstrom, A.D.; Marshall, P.W.; Lonsdale, C.; Cheema, B.S.; Fiatarone Singh, M.A.; Green, S. Resistance training improves fatigue and quality of life in previously sedentary breast cancer survivors: A randomised controlled trial. Eur. J. Cancer Care 2016, 25, 784-794. [CrossRef] [PubMed]

18. Hagstrom, A.D.; Shorter, K.A.; Marshall, P.W.M. Changes in unilateral upper limb muscular strength and emg activity following a 16 week strength training intervention survivors of breast cancer. J. Strength Cond. Res. 2017. [CrossRef] [PubMed]

19. Denham, J.; O’Brien, B.J.; Prestes, P.R.; Brown, N.J.; Charchar, F.J. Increased expression of telomere-regulating genes in endurance athletes with long leukocyte telomeres. J. Appl. Physiol. 2016, 120, 148-158. [CrossRef] [PubMed]

20. Denham, J. Lack of association between pbmc telomere length and endurance exercise. J. Appl. Biomed. 2017, 15, 9-13. [CrossRef]

21. Denham, J.; Marques, F.Z.; Charchar, F.J. Leukocyte telomere length variation due to DNA extraction method. BMC Res. Notes 2014, 7, 877. [CrossRef] [PubMed]

22. Duggan, C.; Risques, R.; Alfano, C.; Prunkard, D.; Imayama, I.; Holte, S.; Baumgartner, K.; Baumgartner, R.; Bernstein, L.; Ballard-Barbash, R.; et al. Change in peripheral blood leukocyte telomere length and mortality in breast cancer survivors. J. Natl. Cancer Inst. 2014, 106, dju035. [CrossRef] [PubMed]

23. Lin, J.; Epel, E.; Blackburn, E. Telomeres and lifestyle factors: Roles in cellular aging. Mutat. Res. 2012, 730, 85-89. [CrossRef] [PubMed]

24. Buxton, J.L.; Das, S.; Rodriguez, A.; Kaakinen, M.; Couto Alves, A.; Sebert, S.; Millwood, I.Y.; Laitinen, J.; O'Reilly, P.F.; Jarvelin, M.R.; et al. Multiple measures of adiposity are associated with mean leukocyte telomere length in the northern finland birth cohort 1966. PLoS ONE 2014, 9, e99133. [CrossRef] [PubMed]

25. Nordfjall, K.; Eliasson, M.; Stegmayr, B.; Melander, O.; Nilsson, P.; Roos, G. Telomere length is associated with obesity parameters but with a gender difference. Obesity 2008, 16, 2682-2689. [CrossRef] [PubMed]

(C) 2018 by the authors. Licensee MDPI, Basel, Switzerland. This article is an open access article distributed under the terms and conditions of the Creative Commons Attribution (CC BY) license (http://creativecommons.org/licenses/by/4.0/). 